\title{
NOUVEL ESSAI DE CLASSIFICATION DES NEMATODES TRICHOSTRONGYLOIDEA
}

\author{
par M.-C. DURETTE-DESSET et A. G. CHABAUD*.
}

RÉSUMÉ. L'essai de classification que nous avons proposé en 1977 groupait à titre provisoire, dans la famille des Amidostomatidae tous les Trichostrongyloidea primitifs (à l'exception des Strongylacanthidae).

L'étude de quelques Nématodes de Reptiles et d'Amphibiens, et surtout celle des parasites de Monotrèmes et de Marsupiaux d'Australie, amènent à dissocier cette famille.

Nous admettons que 2 familles, Amidostomatidae et Strongylacanthidae sont issues d'ancêtres proches des Ancylostomatoidea. Toutes les autres, au contraire, semblent issues des Strongyloidea. Les caractères morphologiques pris en considération permettent d'individualiser trois lignées principales, fondées surtout, chez les formes primitives, sur l'anatomie de la bourse caudale, et, chez les formes spécialisées, sur la structure du synlophe.

L'évolution principale du groupe paraît s'être effectuée à l'ère secondaire dans le Gondwana. Les familles et sous-familles restent très nombreuses principalement en zones australienne et néotropicale.

La faune holarctique, au contraire, bien qu'elle soit extrêmement riche en espèces, paraît restreinte à un petit nombre de familles. Les taxa admis au rang de famille sont : Strongylacanthidae, Amidostomatidae, Dromaeostrongylidae, Trichostrongylidae, Amphibiophilidae, Dictyocaulidae, Molineidae, Mackerrastrongylidae, Nicollinidae, Herpetostrongylidae, Viannaiidae, Ornithostrongylidae, Heligmosomidae, Heligmonellidae.

\section{A new classification of Trichostrongyloidea Nematodes.}

SUMMARY. The preliminary classification that we proposed in 1977 grouped provisionally in the family Amidostomatidae all the primitive Trichostrongyloidea (with the exception of the Strongylacanthidae).

The study of several nematodes from reptiles and amphibians, and above all parasites of Australian monotremes and marsupials, lead us to split up this family.

We believe that two families, Amidostomatidae and Strongylacanthidae evolved from ancestors close to the Ancylostomatoïdea. All the others, however, appear to have evolved from the Strongyloïdea.

The morphological characters considered permit the elucidation of three principal evolutionary lines, recognized for the primitive forms by the anatomy of the caudal bursa and for specialized forms by the structure of the synlophe.

The principal evolution of the group seems to have occurred during the Secondary Era in Gondwanaland. The families and sub-families remain very numerous in the Neotropical and Australian zones.

* Laboratoire des Vers, associé au C.N.R.S. Muséum national d'Histoire naturelle, 43, rue Cuvier, F 75231 Paris Cedex 05.

Accepté le 9 décembre 1980. 
In comparison, the holarctic fauna, despite its richness in species, appears to be restricted to a small number of families. The taxa considered of family rank are: Strongylacanthidae, Amidostomatidae, Dromaeostrongylidae, Trichostrongylidae, Amphibiophilidae, Dictyocaulidae, Molineidae, Mackerrastrongylidae, Nicollinidae, Herpetostrongylidae, Viannaiidae, Ornithostrongylidae, Heligmosomidae, Heligmonellidae.

Nous avons publié en 1977 un essai de classification des Nématodes Trichostrongyloïdes en utilisant très largement les caractères fournis par le synlophe.

Pour la faune australienne, nous indiquions : "Pour les parasites de Marsupiaux australiens, nous adoptons provisoirement les conclusions d'Inglis, 1968, mais la question sera réétudiée prochainement à l'aide du synlophe ».

Grâce à la générosité de nos collègues australiens, nous avons pu effectuer ce travail, et celui-ci mène à des remaniements systématiques plus étendus que ceux auxquels nous nous attendions.

Il est apparu, en effet, que les parasites de Monotrèmes, étonnamment proches de quelques formes reliques que nous connaissons chez différents Amphibiens et Reptiles, semblent constituer un témoin de l'évolution des Nématodes Trichostrongyloïdes au cours de l'ère secondaire. On rencontre déjà, chez ces formes reliques, des tendances évolutives caractéristiques qui s'épanouiront, au cours du Tertiaire, lorsque la superfamille colonisera les Mammifères placentaires.

Cela conduit à une dissociation très poussée de la famille des Amidostomatidae, dans laquelle nous avions groupé, à titre provisoire, toutes les formes que nous considérions comme ancestrales.

L'objet de ce travail est premièrement d'exposer dans ses grandes lignes les hypothèses que nous formulons sur l'évolution du groupe, deuxièmement de formuler les modifications taxonomiques qui en découlent.

\section{Hypothèses générales sur l'évolution du groupe.}

Deux familles sont nettement distinctes des autres Trichostrongyloidea. 1 - Les Strongylacanthidae.

Le genre unique Strongylacantha, qui est parasite de Chiroptères Rhinolophidés, fait, pour les auteurs russes, partie des Ancylostomatoidea, ce qui se justifie à cause de ses puissants crochets intrabuccaux.

Nous préférons cependant le classer parmi les Trichostrongyloidea parce que, en plus de l'ovéjecteur, les épines de la pointe caudale des femelles et l'onchium de la glande oesophagienne dorsale, libre dans la capsule buccale, nous semblent caractéristiques de cette super-famille.

La bourse caudale, de type très primitif, la présence d'une petite capsule buccale, indiquent qu'il s'agit d'une forme archaïque, mais il paraît difficile de préciser de quel ancêtre Ancylostomatoidea ou Strongyloidea ils peuvent dériver. 


\section{2 - Les Amidostomatidae.}

Cette famille correspondait précédemment à l'ensemble des formes primitives pourvues d'une capsule buccale, ainsi qu'aux Mackerrastrongylidae, définis à l'époque par la présence de rameaux supplémentaires sur la côte dorsale.

Le genre type Amidostomum a, en réalité, une structure céphalique bien particulière, qui ne se retrouve dans aucun autre groupe de Trichostrongyloidea. Les papilles céphaliques sont constituées par de longs pédoncules qui s'étendent sur toute la hauteur de la capsule buccale et se terminent au niveau de la bouche (cf. Wertheim et Durette-Desset, 1975, p. 739, fig. 2, H). Ces structures évoquent celles des Globocéphales.

Nous interprétons donc les Amidostomatidae comme un rameau très particulier issu vraisemblablement d'un groupe proche de celui des Globocephalinea (Ancylostomatoidea), n'ayant pas de filiation directe avec les autres Trichostrongyloïdes.

Dans cette conception, la famille se trouve donc limitée au genre type Amidostomum et aux Epomidiostominae qui en dérivent directement comme le montre l'analogie des bourses caudales.

Cette solution rejoint celle qu'avaient proposée les auteurs soviétiques dès 1952 (Skrjabin et coll., 1952).

En dehors de ces deux petites familles aberrantes, l'ensemble de la super-famille des Trichostrongyloidea paraît homogène et issue d'ancêtres proches de Strongyloidea primitifs.

L'évolution se fait par perte de la capsule buccale, par modification de la bourse caudale, par modification de la structure des spicules, qui, le plus souvent, s'allongent et se simplifient, par acquisition d'un ovéjecteur caractéristique et, chez beaucoup d'espèces, par apparition d'un appareil de fixation original : le synlophe. La vésicule céphalique pourrait être un appareil sensoriel spécialisé, destiné au fonctionnement du synlophe, et son existence semble donc liée à ce dernier.

L'analyse de l'évolution des principaux caractères morphologiques conduit aux constatations suivantes :

I - SynLopHe : Il permet de reconnaître trois grands groupes :

A. Synlophe rudimentaire, parfois inexistant.

B. Synlophe généralement bien développé, mais conservant la symétrie bilatérale primitive.

C. Synlophe très spécialisé avec acquisition de nouveaux axes de symétrie, adaptés à l'enroulement du corps le long de sa ligne ventrale gauche.

2 - Capsule buccale : elle disparaît d'emblée dans les groupes A et B, à l'exception du genre relique Amphibiophilus; elle persiste au contraire chez certaines familles du groupe $\mathrm{C}$.

3 - Bourse caudale : Les éléments de classification fournis par l'évolution de la bourse caudale sont les plus difficiles à juger, car celle-ci subit souvent des hyperévo- 
lutions aberrantes chez les formes spécialisées. Il faut donc s'appuyer essentiellement sur les caractéristiques des formes les plus primitives.

On peut alors distinguer :

- groupe A : tendance à la conservation d'un grand lobe dorsal, tendance à l'isolement de la côte 2 ;

- groupe B : conservation de la disposition primitive 2-1-2, c'est-à-dire côtes 2-3 longues, groupées, bien séparées de la côte 4 , généralement courte, elle-même bien séparée des côtes 5-6 groupées ;

- groupe C : évolution rapide de la bourse caudale avec réduction du lobe dorsal et perte de la disposition 2-1-2, (fig. I).

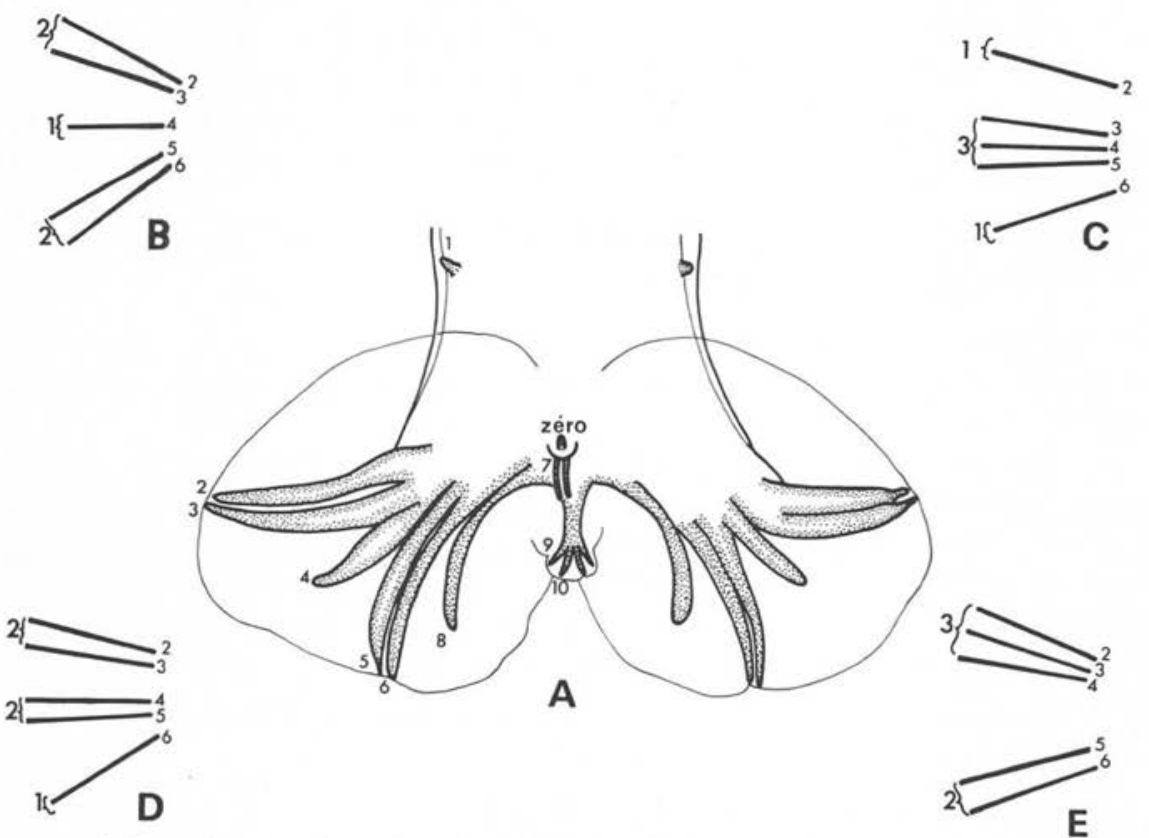

IG. I. - Désignation des côtes bursales (A) et schématisation des types de bourse caudale citées de façon abrégée dans le texte : B, type $2-\mathrm{I}-2$ "Molineus \#, C, type I - 3 - I * Nicollina », D, type 2-2 - I «Ornithostrongylus », E, type 3-2 "Woolleya ».

4 - Spicules : Les spicules nous paraissent avoir plus de valeur spécifique que générique. Cependant, il est possible de remarquer qu'en règle générale le type court et à extrémités distales complexes est assez caractéristique des groupes A et B. Dans le groupe C, au contraire, l'évolution s'est faite rapidement par un allongement du corps du spicule et une simplification de l'extrémité distale.

5 - Pointe caudale de la femelle : Absente dans le groupe A, présente chez les formes primitives du groupe B, présente ou absente selon les lignées dans le groupe C. 6 - Monodelphie : Exceptionnelle dans le groupe A, elle n'apparaît que tardivement 
dans le groupe $\mathrm{B}$ et se manifeste au contraire très précocement au cours de l'évolution du groupe $\mathrm{C}$.

\section{GROUPE A}

Ce groupe comprend une famille primitive : les Dromaeostrongylidae, qui ne s'est bien diversifiée qu'en Australie, et une famille spécialisée : les Trichostrongylidae, qui a divergé assez précocement de la précédente.

Les Dromaeostrongylidae comprennent le genre Paramidostomum, qui reste proche des parasites d'Amphibiens primitifs, tels que Batrachostrongylus, mais s'en distingue surtout par l'isolement et la réduction de la côte 2. A la suite, le genre Dromaeostrongylus, parasite de Ratites australiens, est particulièrement intéressant, car il ressemble à Libyostrongylus, parasite de Ratites éthiopiens, qui fait déjà partie des Trichostrongylidae.

Le genre Peramelistrongylus a de réelles affinités avec Batrachostrongylus (Amphibiophilidae), parasite de Batraciens malais, et pourrait faire transition avec la petite lignée Profilarinema, Filarinema, qui se diversifie chez les Phalangeroidea.

Filarinema a une localisation particulière dans la région pylorique de l'estomac et acquiert un appareil buccal spécialisé.

Les Dromaeostrongylidae sont dépourvus de lèvres ; la capsule buccale est réduite à un anneau ou disparaît ; la côte dorsale est très longue chez les formes primitives, elle est plus courte et profondément divisée chez les formes spécialisées.

A partir de certains Dromaeostrongylidae, paraît naître la sous-famille des Libyostrongylinae. Le genre Libyostrongylus est parasite de Ratites africains et fait transition avec Paralibyostrongylus, qui conquiert les Mammifères Euthériens, en s'inféodant à différents Mammifères archaïques éthiopiens.

L'évolution de la grande famille des Trichostrongylidae et de ses différentes sousfamilles, par adaptation aux Lagomorphes, puis aux Artiodactyles, a été détaillée dans le travail de 1977.

\section{Groupe B}

Une forme ancestrale dont la morphologie reste très proche de celle d'un Strongyloidea est représentée par le genre Amphibiophilus, parasite d'Amphibiens en Afrique et en Asie. Il est groupé avec Batrachostrongylus dans une famille particulière, les Amphibiophilidae.

Le groupe particulier des Dictyocaulidae paraît prendre naissance à partir d'un ancêtre peu éloigné d'Amphibiophilus.

La place des Dictyocaules (parasites des poumons d'Ongulés et de Primates) est un sujet de controverse, puisque, par leur morphologie et leur biologie, ils sont intermédiaires entre Trichostrongyloidea et Metastrongyloidea. La connaissance des genres Mertensinema et Borrellostrongylus, parasites d'Amphibiens et de Reptiles en différentes régions du monde, semble permettre d'éclaircir le problème car leurs caractéristiques morphologiques (bourse caudale ronde avec côtes 5-6 proches ou 
fusionnées, côte dorsale grande et profondément divisée, femelle sans pointe caudale, synlophe absent) font transition entre la morphologie d'un Trichostrongyloïde primitif et celle d'un Dictyocaule.

Nous avons donc groupé les genres cités plus haut, parasites d'Amphibiens et Reptiles, dans la sous-famille des Mertensinematinae, placée à la base de la famille des Dictyocaulidae, les Dictyocaulinae, parasites d'Ongulés et de Primates constituant la deuxième sous-famille.

En dehors des Dictyocaules, le groupe B, issu d'ancêtres proches d'Amphibiophilus, est constitué de deux familles : d'une part, les Molineidae, parasites d'Amphibiens, de Reptiles et de Mammifères Euthériens, et, d'autre part, les Mackerrastrongylidae, qui parasitent les Monotrèmes et Marsupiaux australiens.

La plupart des Mackerrastrongylidae conservent une forte dent dorsale, mais la morphologie des deux familles reste très proche.

I - Les Molineidae sont divisés en quatre sous-familles:

a - Les Molineinae : Ils sont parasites d'Amphibiens, d'Insectivores Tenrecoidea, - de Carnivores, de Chiroptères du Vieux Monde, de Tupaiidés, de Primates, de Pholidotes, de Tubulidentés et de Sciuridés). Ils ont donc conquis l'ensemble des Vertébrés qui ont eu leur phase d'expansion en zones éthiopienne, orientale et holarctique, au début du Tertiaire.

b - Les Anoplostrongylinae: Ils se distinguent des précédents surtout par une élaboration du synlophe. Ce sont des parasites de Chiroptères et de Xénarthres néotropicaux.

c - Les Nematodirinae : Leur côte dorsale est divisée dès la base en deux racines distinctes et ils acquièrent une formation néodonte. Ils s'adaptent aux Lagomorphes, puis aux Ruminants.

d - Les Ollulalinae : Ils sont souvent exclus des Trichostrongyloïdes à cause de leur viviparité et de leur cycle évolutif particulier. La morphologie des adultes (bourse caudale, spicules, extrémité céphalique) n'offre pas de difficultés particulières pour les rattacher à la famille des Molineidae.

2 - Les Mackerrastrongylidae sont constitués de deux sous-familles:

a - Les Tachynematinae (genres Tachynema, Tasmanema, Zaglonema) : Ils groupent les formes les plus primitives et sont exclusivement parasites de Monotrèmes. Ils conservent une capsule buccale relativement bien marquée, mais n'ont plus les six lèvres primitives. Ils paraissent avoir donné naissance aux Mackerrastrongylinae.

$\mathrm{b}$ - Les Mackerrastrongylinae : Ils ressemblent beaucoup aux Molineinae, mais possèdent un rameau accessoire supplémentaire sur la côte dorsale. Ce sont des parasites de Marsupiaux australiens primitifs (Dasyuroidea et Perameloidea).

Groupe C

Chacune des trois grandes lignées qui constituent ce groupe est caractéristique d'une région biogéographique déterminée. 
- La première branche, la plus primitive, très remarquable par la conservation d'une forte capsule buccale, est australienne. Elle comprend deux familles :

I - Les Nicollinidae : ce sont des parasites de Monotrèmes. Ils comprennent également le genre Batrachonema, parasite d'un Amphibien de Malaisie, dont la morphologie n'est pas plus primitive que celle des autres genres; il dérive donc peut-être secondairement de la faune des Monotrèmes. La famille comprend, en outre, un genre un peu aberrant, Copemania, parasite de Marsupiaux.

2 - Les Herpetostrongylidae, avec deux sous-familles :

a - Les Globocephaloidinae : L'analyse morphologique des Globocephaloidinae a été faite par Inglis, 1968 et par Beveridge, 1979. Nous sommes en accord avec leurs conclusions pour estimer qu'il s'agit d'une forme hautement spécialisée, mais issue d'une souche australienne.

La spécialisation des Globocephaloidinae est si poussée que son origine est difficile à préciser. Les Dromaeostrongylinae ne peuvent constituer cette origine, puisqu'ils évoluent rapidement vers l'atrophie des structures céphaliques. Entre les Mackerrastrongylidae, les Nicollinidae, les Herpetostrongylidae, nous choisirons de préférence une origine commune avec ces derniers.

En effet, l'évolution de la bourse caudale avec des côtes 2 et 3 associées à la base en un pédoncule commun et le fort développement du trident latéral sont des évolutions que l'on rencontre plutôt chez les Herpetostrongylidae (Dessetostrongylus par exemple) que dans les autres familles.

Nous considérons donc les Globocephaloidinae comme une sous-famille des Herpetostrongylidae, mais cette décision systématique reste, bien entendu, très aléatoire et n'est adoptée qu'à titre provisoire.

b - Les Herpetostrongylinae sont des parasites de Reptiles en Australie et dans la zone orientale, et de Marsupiaux depuis les Dasyuroidea jusqu'aux Phalangeroidea. Ils se différencient des Nicollinidae par la disposition des côtes bursales, qui est de type 1-3-1 chez ces derniers, tandis que chez les Herpetostrongylinae, les côtes 2, 3, 4, sont grandes, généralement espacées et s'opposent aux côtes 5, 6, petites et groupées.

- La seconde branche de ce groupe est néotropicale. Elle comprend également deux familles :

I - Les Viannaiddae : Ils sont parasites de Marsupiaux américains et conquièrent certains Rongeurs Caviomorphes. A l'inverse des autres familles du groupe C, les Viannaiidae conservent une bourse caudale de type 2-1-2, donc de type primitif.

2 - Les Ornithostrongylidae : Ils comprennent deux sous-familles :

a - Les Inglamidinae: Ils sont connus actuellement par une seule espèce récoltée chez un Cricétidé du Chili et qui est vraisemblablement, en réalité, un parasite primitif de Marsupial, comme l'atteste la conservation de la structure très primitive de l'extrémité céphalique. 
b - Les Ornithostrongylinae : Ils sont parasites d'Oiseaux et de Chiroptères en Amérique du Sud, de Rongeurs archaïques (Geomyoidea en Amérique du Nord), avec quelques représentants parasites d'Oiseaux ou de Chiroptères dans l'Ancien Monde. La bourse caudale est de type 2-2-1, et le synlophe est symétrique par rapport à l'axe frontal.

Vexillata, qui était interprété précédemment comme un Viannaiinae hyperévolué, paraît, en réalité, pouvoir être rapproché des Ornithostrongylinae à cause de la disposition des côtes bursales.

- La troisième branche, a pris son extension dans les régions éthiopienne, orientale et holarctique, et ne déborde que secondairement dans les zones australienne et néotropicale.

Elle n'explose que tardivement, probablement à l'Eocène, car les formes les plus primitives sont connues chez les Insectivores Soricoïdea pour l'une des familles, et les Insectivores Talpoidea pour l'autre famille.

D'un point de vue morphologique, l'ensemble s'oppose aux formes australiennes, car il y a atrophie des structures céphaliques. La bourse caudale n'a pas la disposition 2-1-2 des Viannaiidae; enfin, ils s'opposent aux Ornithostrongylidae, parce qu'à de très rares exceptions près, ils sont monodelphes. Ils apparaissent donc à tous points de vue comme plus évolués que les formes correspondantes en Australie et en Amérique du Sud.

Ils sont divisés en deux familles :

I - Les Heligmosomidae : La pointe des crêtes ou des arêtes du synlophe est orientée de la droite vers la gauche. La femelle possède une épine caudale sur la queue. La bourse caudale est de type 3-2. Ce sont des parasites d'Insectivores Soricoidea, de Lagomorphes et de Rongeurs Myomorphes de l'Ancien Monde et de la région néarctique.

2 - Les Heligmonellidae: Leur synlophe présente un axe d'orientation des arêtes obliques par rapport à l'axe frontal (sauf chez les formes les plus évoluées). La queue des femelles est dépourvue d'épines. La bourse caudale est de type 2-2-1. Ce sont des parasites d'Insectivores Talpoidea, de Lagomorphes et de Rongeurs Phiomorphes, Caviomorphes et Myomorphes. La division des Heligmonellidae en 4 sous-familles et l'évolution particulière à chacune d'entre elles sont détaillées dans le travail de 1977 .

\section{Taxonomie proposée.}

I - Strongylacanthidae (Yorke et Maplestone, 1926, sub. fam.) Chabaud, 1960.

Genre unique : Strongylacantha Van Beneden, 1873.

Hôtes et répartition géographique : Chiroptères Rhinolophidés.

2 - Amidostomatidae (Travassos, 1919 sub. fam.) Baylis et Daubney, 1926.

a - Amidostomatinae Travassos, 1919.

Genre type unique : Amidostomum Railliet et Henry, 1909.

Hôtes et répartition géographique : Oiseaux. Cosmopolites. 
b - Epomidiostomatinae Skrjabin et Schulz, 1937.

Genre type : Epomidiostomum Skrjabin, 1915.

Autre genre : Pseudamidostomum Boulenger, 1926.

Hôtes et répartition géographique : Oiseaux. Cosmopolites.

3 - Dromaeostrongylidae (Skrjabin et Schulz, 1937, tribu) n. fam.

Genre type : Dromaeostrongylus Lubimov, 1933.

Autres genres : Filarinema Mönnig, 1929 (= Asymmetricostrongylus Nagaty, 1932) ;

Paramidostomum Freitas et Mendonça, 1950 ; Peramelistrongylus Mawson, 1960 ;

Profilarinema Durette-Desset et Beveridge, 1981.

Hôtes et répartition géographique : Oiseaux brésiliens, Oiseaux-Ratites et Marsupiaux d'Australie.

4 - Trichostrongylidae (Leiper, 1908, sub. fam.) Leiper, 1912.

a - Libyostrongylinae Durette-Desset et Chabaud, 1977.

Genre type : Libyostrongylus Lane, 1923.

Autres genres : Cnizostrongylus Chabaud, Durette-Desset et Houin, 1967 ; Moguranema Yamaguti, 1941. Obeliscoides Graybill, 1924 (=Obeliscus Graybill, 1923) ; Paralibyostrongylus Ortlepp, 1939 (= Baylisiella Skrjabin et Schulz, 1937); Pararhabdonema Kreis, 1945 ; Pseudostertagia Orloff, 1933.

Hôtes et répartition géographique : Oiseaux-Ratites et Mammifères archaïques paléarctiques et éthiopiens; Lémuriens et Rongeurs malgaches; Antilocapra en région néarctique et Lagomorphes.

b - Cooperiinae (Skrjabin et Schulz, 1937, tribu) Skrjabin et Schikhobalova, 1952.

Genre type : Cooperia Ransom, 1907.

Autres genres : Chabaudstrongylus Durette-Desset et Denké, 1978: Cooperioides Daubney, 1933 ; Gazellostrongylus Yeh, 1956 ; Impalaia Mönnig, 1923 (= Anthostrongylus Croveri, $1929=$ Minutostrongylus Le Roux, 1936) ; Megacooperia Khalil et Gibbons, 1976; Ortleppstrongylus Durette-Desset, 1970, Paracooperia Travassos, 1935 (= Schwartziella Le Roux, 1936).

Hôtes et répartition géographique : Bathyergidés et Ruminants, essentiellement de l'Ancien Monde.

c - Graphidiinae Travassos, 1937.

Genre type : Graphidium Railliet et Henry, 1909.

Autres genres : Graphidiella Olsen, 1948; Hyostrongylus Hall, 1921 ; Parostertagia Schwartz et Alicata, 1933.

Hôtes et répartition géographique : Lagomorphes holarctiques et éthiopiens, Tragulidés et Suidés. Cosmopolites.

d - Ostertagiinae (Skrjabin et Schultz, 1937, tribu) Sarwar, 1956.

Genre type : Ostertagia Ransom, 1907 (= Grunheria Sarwar, 1956 = Ostertagiella Andreeva, 1956.) 
Autres genres : Apteragia Jansen, 1958 (=Spiculopteroïdes Jansen, 1958) ; Bergheia Drozdz, 1965 ; Camelostrongylus Oloff, 1933 ; Capreolagia Schulz, Andreeva et Kadenazii, 1953 ; Grosspiculagia (Orloff, 1933) Sarwar, 1956 ; Longistrongylus Le Roux, 1931 (= Bigalkea Mönnig, 1931, = Bigalkenema Ortlepp, 1963, = Kobusinema Ortlepp, 1963) ; Marshallagia (Orloff, 1933) Travassos, 1937 ; Mazamastrongyhus Cameron, 1935 ; Muflonagia Schulz, Andreeva et Kadenazii, 1953 ; Orloffia Drozdz, 1965 ; Ostertagiana, Dikov, 1963 ; Ostertamia Dikov, 1963 ; Paramecistocirrus Roetti, 1941 ; Rinadia Grigorian, 1951; Sarwaria Drozdz, 1965; Skrjabinagia (Kassimov, 1942) Altaev, 1952 (=Sjobergia Sarwar, 1956) ; Spiculopteragia Orloff, 1933 (= Altaevia Sarwar, 1957, = Mazamastrongylus Cameron, 1935) ; Teladorsagia Andreeva et Satubaldin, 1953 (=Stadelmannia, 1956).

Hôtes et répartition géographique : Ruminants essentiellement de l'Ancien Monde.

e - Trichostrongylinae Leiper, 1912.

Genre type : Trichostrongylus Loos, 1905 (=Buckleya Sarwar, 1956, = Cobbostrongylus Sarwar, 1956, = Gilesia Sarwar, 1956).

Autres genres: Graphidioïdes Cameron, 1923 ; Travassosius Khalil, 1922.

Hôtes et répartition géographique : Oiseaux, Ruminants et Lagomorphes du monde entier ; Rongeurs " anciens » du Nouveau Monde.

f - Haemonchinae (Skrjabin et Schulz, 1937, tribu) Skrjabin et Schulz, 1952.

Genre type : Haemonchus Cobb, 1898.

Autres genres: Ashworthius Le Roux, 1930 ; Biogastranema Rohrbacher et Ehrenford, 1954 ; Boehmiella Gebauer, 1932 ; Leiperiatus Sandground, 1930 ; Mecistocirrus Railliet et Henry, 1912.

Hôtes et répartition géographique : Ruminants essentiellement de l'Ancien Monde. 5 - Amphibiophilidae n. fam.

Genre type : Amphibiophilus Skrjabin, 1916.

Autre genre : Batrachostrongylus Yuen, 1963.

Hôtes et répartition géographique : Amphibiens et Reptiles d'Afrique et d'Asie.

6 - Dictyocaulidae (Skrjabin, 1933, s. fam.) Skrjabin, 1941.

a - Mertensinematinae Sharpilo, 1976.

Genre type : Mertensinema Sharpilo, 1976.

Autre genre : Borrellostrongylus Gutierrez, 1945 (= Parabatrachostrongylus Tantalean et Naupay, 1974.)

Hôtes et répartition géographique : Amphibiens paléarctiques et néotropicaux, Reptiles de Bornéo.

b - Dictyocaulinae Skrjabin, 1933.

Genre type : Dictyocaulus Railliet et Henry, 1907 (= Micrurocaulus Skrjabin, 1934). 
Autres genres : Bronchonema Mönnig, 1932 ; Otostrongylus de Bruyn, 1933 (= Kutassicaulus Skrjabin, 1933; = Kutastrongylus Skrjabin, 1933).

Hôtes et répartition géographique : Ungulés, Pinnipèdes. Cosmopolites. (Bronches). 7 - Molineidae (Skrjabin et Schulz, 1937, s. fam.) Durette-Desset et Chabaud, 1977.

a - Molineinae Skrjabin et Schulz, 1937.

Genre type : Molineus Cameron, 1923 (= Microstrongylus Cameron, 1927 ; = Nematostrongylus Cameron, 1928 ; = Tenuostrongylus Le Roux, 1933).

Autres genres : Angulocirrus Biocca et Le Roux, 1956 ; Dollfusstrongylus Quentin, 1970, Hepatojarakus Yeh, 1955 ; Molinostrongylus Skarbilowitch, 1934 ; Nochtia Travassos et Vogelsang, 1929 ; Nycteridostrongylus Baylis, 1930 ; Oswaldocruzia Travassos, 1917 ; Pithecostrongylus Lubimov, 1930 (= Hooperstrongylus Lie Kian Joe et Ow Yang, 1963) ; Poekilostrongylus Schmidt et Whittaker, 1975 ; Schulzia Travassos, 1937 ; Trichochenia Kou, 1958 (= Manistrongylus Baer, 1959, = Mani strongylus Cameron et Myers, 1960, = Pholidostrongylus Baer, 1959) ; Shattuckius Sandground, 1938 ; Trichoskrjabinia Travassos, 1937 ; Tupaiostrongylus Dunn, 1963 ; Typhlopsia Barus et Otero, 1976.

Hôtes et répartition géographique : Batraciens, Tortues, Insectivores Tenrecoidea, Carnivores, Chiroptères (du vieux monde), Tupaiidés, Primates, Pholidotes, Tubulidentés et Sciuridés. Cosmopolites.

b - Ollulaninae Hall, 1916.

Genre type unique : Ollulanus Leuckart, 1865.

Hôtes et répartition géographique : Mammifères. Cosmopolites.

c - Anoplostrongylinae Chandler, 1938.

Genre type : Anoplostrongylus Boulenger, 1926.

Autres genres : Adolpholutzia Travassos, 1935 ; Biacantha Wolfgang, 1954 ; Bidigiticauda Chitwood, 1938; Bradypostrongylus Price, 1928 (= Pintoia Travassos, 1928) ; Brevigraphidium Freitas et Mendonça, 1960; Caenostrongylus Lent et Freitas, 1938 ; Cheiropteronema Sandground, 1929 ; Dasypostrongylus Travassos, 1935 ; Delicata Travassos, 1935 ; Didactyluris Freitas et Dobbin, 1960 ; Filicapitis Travassos, 1949; Fontesia Travassos, 1928; Graphidiops Lent et Freitas, 1938 ; Histiostrongylus Molin, 1861 ; Maciela Travassos, 1935 ; Moennigia Travassos, 1935 (= Pintonema Travassos, 1935, = Pulchrostrongylus Travassos, 1935) ; Neohistiostrongylus Barus et Rysavy, 1971 ; Paragraphidium Freitas et Mendonça, 1959 ; Parahistiostrongylus Vigueras, 1941 ; Spinostrongylus Travassos, 1935 ; Torrestrongylus Vigueras, 1935 ; Trichohelix Ortlepp, 1922 ; Tricholeiperia Travassos, 1935 ; Trifurcata (Schulz, 1926).

Hôtes et répartition géographique : Chiroptères et Xénarthres néotropicaux (une espèce chez un Chiroptère de l'Ancien Monde et deux espèces chez des Cricétidés néotropicaux). 
d - Nematodirinae Skrjabin et Orloff, 1934.

Genre type : Nematodirus Ransom, 1907.

Autres genres : Lamanema Becklund, 1963 ; Murielus Dikmans, 1939 ; Nematodirella Yorke et Maplestone, 1926 (= Microcephahus Romanovitch, 1915) ; Nematodiroides Bernard, 1967 ; Rauschia Durette-Desset, 1979.

Hôtes et répartition géographique : Lagomorphes et Ruminants. Cosmopolites.

8 - Mackerrastrongylidae (Inglis, 1968, s. fam.) n. fam.

a - Tasmanematinae n. sub. fam.

Genre type : Tasmanema Durette-Desset et Beveridge, 1981.

Autres genres : Tachynema Durette-Desset et Beveridge, 1981 ; Zaglonema DuretteDesset et Beveridge, 1981.

Hôtes et répartition géographique : Tachyglossus (Australie), Zaglossus (Nouvelle Guinée).

b - Mackerrastrongylinae Inglis, 1968.

Genre type : Mackerrastrongylus Mawson, 1960.

Autres genres : Asymmetracantha Mawson, 1960; Sprattelus Durette-Desset et Beveridge, 1981 ; Tetrabothriostrongylus Mawson, 1960.

Hôtes et répartition géographique : Marsupiaux d'Australie (Dasyuroïdea et Perameloïdea).

9 - Nicollinidae (Skrjabin et Schulz, 1937, tribu) n. fam.

Genre type : Nicollina (Baylis, 1930).

Autres genres : Batrachonema Yuen, 1965 ; Copemania Durette-Desset et Spratt, 1981.

Hôtes et répartition géographique : Amphibiens de Malaisie, Monotrèmes, Marsupiaux d'Australie (Dasyuroïdea).

io - Herpetostrongylidae (Skrjabin et Schulz, 1937, s. fam.) n. fam.

a - Globocephaloidinae Inglis, 1968.

Genre type : Globocephaloides Yorke et Maplestone, 1926.

Autre genre : Amphicephaloides Beveridge, 1979.

Hôtes et répartition géographique : Marsupiaux Macropodidae, Australie.

b - Herpetostrongylinae Skrjabin et Schulz, 1937.

Genre type : Herpetostrongylus Baylis, 1937.

Autres genres : Austrostrongylus Chandler, 1924; Beveridgiella Humphery Smith, 1981 ; Dessetostrongylus Humphery Smith, 1981 ; Nasistrongylus Durette-Desset et Beveridge, 1981; Paraustrostrongylus Mawson, 1973; Patricialina Inglis, 1968 ; Vaucherus Durette-Desset, 1981 ; Woolleya Mawson, 1973. 
Hôtes et répartition géographique : Reptiles de Malaisie, des Indes. Reptiles et Marsupiaux australiens.

i - Viannaimae (Neveu-Lemaire, 1934, s. fam.) n. fam.

Genre type : Viannaia Travassos, 1914 (=Philostrongylus Wolfgang, 1951).

Autres genres : Hoineffia Diaw, 1977 ; Oswaldonema Travassos, 1927 (= Heligmoskrjabinia Freitas et Lent, 1937) ; Travassostrongylus Orloff, 1933 (= Camerostrongylus Wolfgang, 1951) ; Viannella Travassos, 1919 (= Avellaria Freitas et Lent, 1934, = Brevispiculoides Ortlepp, 1939).

Hôtes et répartition géographique : Marsupiaux, Rongeurs Cavioïdea (Caviidés, Hydrochoeridés) et Chinchilloidea (Chinchillidés, Cuniculidés) néotropicaux.

12 - Ornithostrongylidae (Travassos, 1937 s. fam.) n. fam.

a - Inglamidinae Durette-Desset, Diaw et Murua, 1976.

Genre type unique : Inglamidum Durette-Desset, Diaw et Murua, 1976.

Hôtes et répartition géographique : Rongeurs Cricétidés du Chili.

b - Ornithostrongylinae Travassos, 1914.

Genre type : Ornithostrongylus Travassos, 1914.

Autres genres : Allintoshius Chitwood, 1937 (= Parallintoshius Araujo, 1940); Columbostrongylus Puylaert, 1968 ; Lutznema Lent et Freitas, 1934 ; Ornithonema Travassos, 1935 ; Oswaldostrongylus Lent et Freitas, 1934 ; Vexillata Hall, 1916.

Hôtes et répartition géographique : Oiseaux Cosmopolites, Chiroptères d'Amérique (quelques espèces du Vieux Monde), Tupaia de Bornéo, Rongeurs Geomyoidea d'Amérique.

I3 - Heligmosomidae (Travassos, 1914, s. fam.) Cram, 1927.

Genre type : Heligmosomum Railliet et Henry, 1909.

Autres genres : Citellinema Hall, 1916 (=Warrenius Hall, 1916) ; Citellinoides Dikmans, 1939; Heligmosomoides Hall, 1916 (= Nematospira Walton, 1923; $=$ Nematospiroides Baylis, 1926, = Paranematospira Sprehn, 1935, = Sincosta Roé, 1929) ; Longistriata Schulz, 1926 (= Longistrioides Yeh, 1958, ? Neoheligmonoides Sadovskaja, 1952) ; Ohbayashinema Durette-Desset, 1974 ; Suncinema Durette-Desset, 1973.

Hôtes et répartition géographique : Insectivores Soricoidea, Lagomorphes, Sciuromorphes, Rongeurs Myomorphes de l'Ancien Monde et de la région néarctique.

I4 - Heligmonellidae (Skrjabin et Schikhobalova, 1952, tribu) Durette-Desset et Chabaud, 1977.

a - Heligmonellinae (Skrjabin et Schikhobalova, 1952, tribu) Durette-Desset, 1971.

Genre type : Heligmonella Mönnig, 1927. 
Autres genres : Paraheligmonella Durette-Desset, 1971; Tricholinstowia Travassos, 1937 (= Morganiella Travassos, 1937, = Morganostrongylus Fahmy, 1956). Xericola Durette-Desset, 1974.

Hôtes et répartition géographique : Insectivores Talpoidea holarctiques, Lagomorphes de l'Ancien Monde et néarctiques, Rongeurs archaïques.

b - Pudicinae (Skrjabin et Schikhobalova, 1952, tribu) Durette-Desset, 1971.

Genre type : Pudica Travassos et Darriba, 1929.

Autres genres : Evandroïa Travassos, 1937; Heligmostrongylus Travassos, 1917 ( = Fuellebornema Travassos et Darriba, 1929, = Heligmodendrium Travassos, 1937, = Squamastrongylus Travassos, 1937, = Stunkardionema Arnold, 1941) ; Pseudoheligmosomum Travassos, 1937 ; Sciurodendrium Durette-Desset, 1971. Trichotravassosia Lent et Freitas, 1938.

Hôtes et répartition géographique : Caviomorphes Chinchilloidea (Dasyproctidés), Octodontoidea (Echimyidés, Capromyidés, Myocastoridés), Erethizontoidea (Erethizontidés), Sciuridés, (une espèce chez un Cricétidé et une autre chez un Lagomorphe) néarctiques et néotropicaux.

c - Brevistriatinae Durette-Desset, 1971.

Genre type : Brevistriata Travassos, 1937.

Autres genres : Calypsostrongylus Schmidt, Myers et Kuntz, 1967; Cordicauda Durette-Desset, 1971; Fissicauda Durette-Desset et Krishnasamy, 1977; Kuala Durette-Desset et Krishnasamy, 1977 ; Metheligmonella Durette-Desset, 1971 ; Paraheligmonina (Ortlepp, 1939) (= Heligmobaylisia Mawson, 1961); Quentinstrongylus Durette-Desset, 1969 ; Srivastavanema (Singh, 1962).

Hôtes et répartition géographique : Rongeurs " anciens ": Hystricidés, Sciuridés, Gliridés de l'Ancien Monde. Hôtes de capture : Tragulidés et Muridés de l'Ancien Monde.

d - Nippostrongylinae Durette-Desset, 1971.

Genre type : Nippostrongylus Lane, 1923 (= Austroheligmonema Mawson, 1961).

Autres genres : Boreostrongylus Durette-Desset, 1971; Hassalstrongylus DuretteDesset, 1971 ; Heligmonina Baylis, 1928 (= Heligmospiroides Ortlepp, 1939, = Trichobaylisia Travassos, 1937); Heligmonoides Baylis, 1928 ; Hypocristata Durette-Desset, 1971; Mammanidula Sadovskaja, 1952 (= Mammaniduloides Ohbayashi, Orihara et Fujimaki, 1968, = Mammolongistriata Dubinin, 1953, = Mastonema Mawson, 1961); Neoheligmonella Durette-Desset, 1971; Odilia Durette-Desset, 1973 (=Austrostrongylus sensu Durette-Desset, 1971) ; Orientostrongylus Durette-Desset, 1970; Stilestrongylus Freitas, Lent et Almeida, 1937 (= Mirandaia Travassos, 1937); Tenorastrongylus Durette-Desset, 1970.

Hôtes et répartition géographique : Rongeurs "récents ": Gerbillidés, Muridés, Arvicolidés du monde entier et Cricétidés du nouveau monde. 


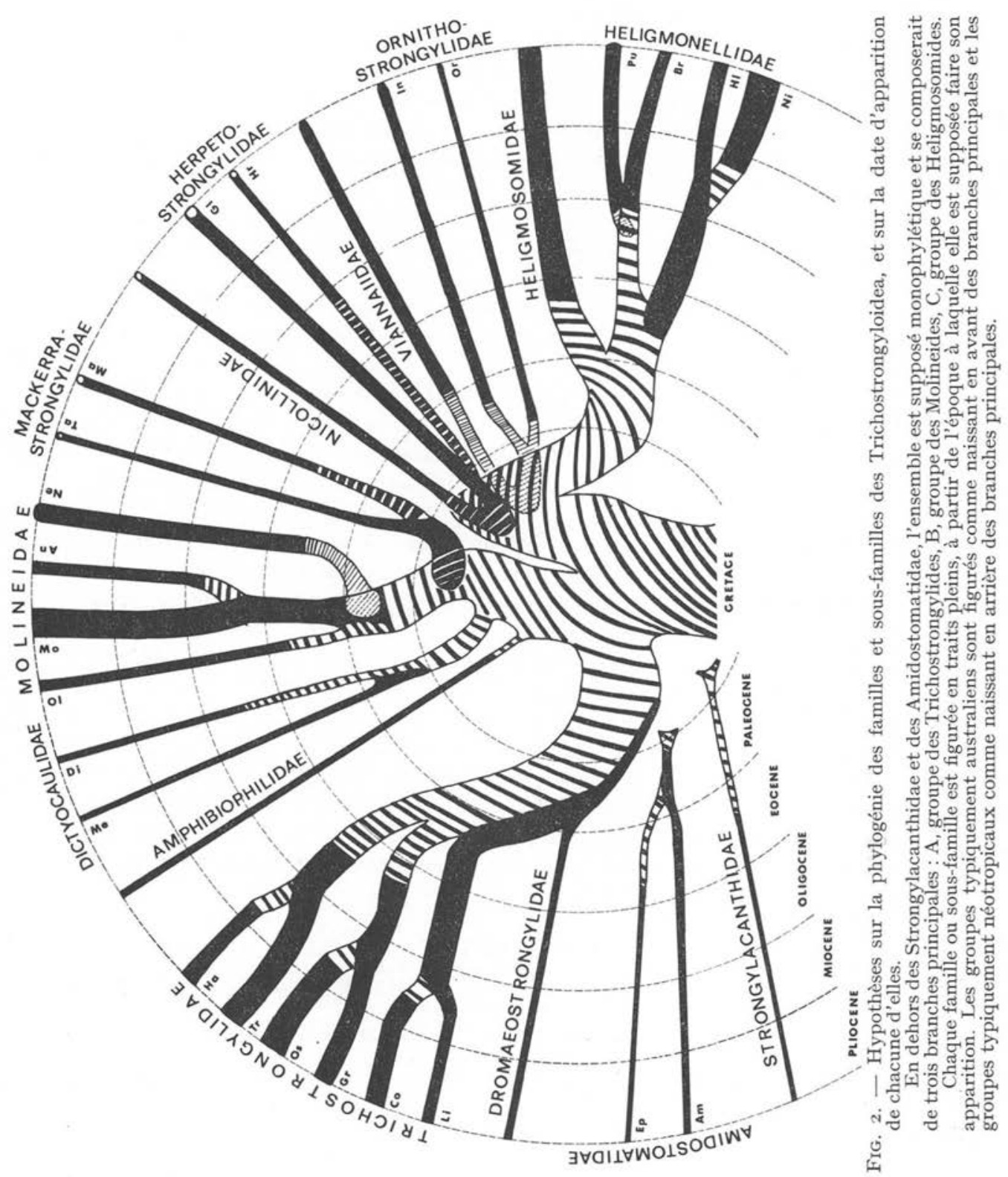




\section{Conclusion}

Il est intéressant de constater que le spectre d'hôtes des familles et sous-familles définies ci-dessus ne groupe pas d'animaux liés par leurs affinités zoologiques.

$\mathrm{Au}$ contraire, le point commun entre les différents hôtes caractéristiques de telle ou telle famille ou sous-famille de Trichostrongyloidea semble être la période géologique à laquelle sont apparus des animaux peu différents des hôtes actuels.

Ceci nous conduit à formuler des hypothèses sur la date d'apparition des familles et sous-familles qui sont indiquées schématiquement sur la figure 2 .

D'un point de vue biogéographique, les Strongylacanthidae et les Amidostomatidae n'ont pas d'intérêt particulier, car ce sont des parasites de Chiroptères ou d'Oiseaux cosmopolites.

Les familles issues des Strongyloidea sont, au contraire, remarquables :

Toutes les formes ancestrales et un bon nombre de familles ou sous-familles sont typiquement gondwaniennes.

La conquête de la Laurasie et des Mammifères Euthériens paraît s'être effectuée par un sévère filtrage, qui, en dépit du nombre extraordinaire d'espèces de la faune actuelle, limite assez étroitement le nombre des familles holarctiques. Nous rencontrons, en effet, essentiellement dans cette région les Trichostrongylidae (parasites cosmopolites des Ruminants issus des parasites de Lagomorphes) et trois grandes familles. 1 - Les Molineidae : les parasites morphologiquement les plus primitifs chez les Mammifères placentaires sont les Molineus des Insectivores Tenrecoidea.

2 - Les Heligmosomidae : les parasites morphologiquement les plus primitifs sont les Suncinema des Insectivores Soricoidea.

3 - Les Heligmonellidae : les parasites morphologiquement les plus primitifs sont les Tricholinstowia des Insectivores Talpoidea.

En s'appuyant sur le fait que les Insectivores et les Marsupiaux sont les seuls Mammifères déjà représentés à la fin du secondaire par des animaux peu différents de ceux de la faune actuelle, l'on peut supposer que l'essentiel de l'évolution des Trichostrongyloidea était réalisée dès le début du Tertiaire, mais la puissante évolution du groupe se manifeste actuellement par l'explosion de genres et d'espèces de certaines familles, particulièrement chez les Marsupiaux australiens, les Rongeurs Arvicolidae et les Bovidae.

\section{BIBLIOGRAPHIE}

Beveridge I. : A review of the Globocephaloidinae Inglis (Nematoda : Amidostomatidae) from Macropodid Marsupials. Aust. J. Zool., 1979, 27, I5I-I75.

Durette-Desset M.-C., Chabaud A. G. : Essai de classification des Nématodes Trichostrongyloidea. Ann. Parasitol. Hum. Comp., 1977, 52, 539-558.

INGLIs W. G. : The geographical and evolutionary relationships of Australian trichostrongyloid parasites and their hosts. J. Linn. Soc. (Zool.), 1968, 47, 312, 327-347.

Skrjabin K. I., Schikhobalova N. P., Schulz R. S., Popova T. I., Boev S. N., Deliamure S. L. : Opredelitel Parasit. Nematode vol. III. Strongyliati, 1952. Akad. Science, édit. Moscou, 890 p.

Wertheim G., Durette-Desset M.-C. : Helminthes de Mammifères et d'Oiseaux d'Israël - VI. La taxonomie et l'écologie des Nématodes Trichostrongyloïdes. Ann. Parasitol. Hum. Comp., I975, 50, 735-762. 